\title{
BOGDANOV-TAKENS BIFURCATIONS IN THREE COUPLED OSCILLATORS SYSTEM WITH ENERGY PRESERVING NONLINEARITY
}

\author{
Livia Owen ${ }^{1}$ And J.M. Tuwankotta ${ }^{2}$ \\ ${ }^{1}$ Parahyangan Catholic University, \\ Jl. Ciumbuleuit 94, Bandung 40141, West Java, Indonesia, \\ livia.owen@unpar.ac.id \\ ${ }^{2}$ Institut Teknologi Bandung, \\ Jl. Ganesha 10, Bandung 40132, West Java, Indonesia, \\ theo@math.itb.ac.id
}

\begin{abstract}
In this paper a system of three nonlinearly coupled oscillators is studied. The linear frequencies of these oscillators are 1,2 and $\varepsilon$ with $\varepsilon \ll 1$. The nonlinearity in the system is assumed to be energy preserving, where the energy is represented by the distance to the origin. We have applied the normal form theory by means of the averaging method, to construct an $O(\varepsilon)$-approximation for the system, on a time-scale of $\frac{1}{\varepsilon}$.

We begin our analysis of the normal form by looking at the unperturbed system, i.e. the normal form for $\varepsilon=0$. We found the saddle-node, pitchfork and homoclinic bifurcations using the classical Lagrange method. To our knowledge, this is a new application of the method in bifurcation theory. We proceed with turning on the perturbation, i.e setting $\varepsilon \neq 0$, and analyzing the bifurcations in the system. Here, we found two saddle-node curves which meet in a cusp bifurcation point. Moreover, we found a Hopf line which is tangent to one of the fold line, which gives us the Bogdanov-Takens bifurcation: a codimension two bifurcation. In the neighborhood of that point, we show homoclinic bifurcation, to give indications of the dynamics. Key words: Normal Form Theory, averaging method, Cusp bifurcation, BogdanovTakens bifurcation.
\end{abstract}

